\title{
LORENTZIAN FORMS FOR THE LEECH LATTICE
}

\author{
BY J. H. CONWAY AND N. J. A. SLOANE
}

\begin{abstract}
Using recent results about holes in the Leech lattice we establish some Lorentzian constructions for that lattice.
\end{abstract}

Some years ago the first author and R. T. Curtis showed by a detailed (and unpublished) calculation that the set of points of the Lorentzian integer lattice $\mathbf{Z}^{24,1}$ which are perpendicular to the vector

$$
t=(3,5,7, \ldots, 45,47,51 \mid 145)
$$

is a copy of the Leech lattice. Our recent work $[1,3]$ on holes in the Leech lattice enables us to give a short proof of this fact. We work instead in the hyperplane of vectors $v \in \mathbf{Z}^{24,1}$ with $v \cdot t=-2$, and observe that this contains all the points mentioned in Figure 1. Two points in the figure are joined by an edge if they are distant $\sqrt{6}$, all other pairs being distant $\sqrt{4}$ apart. Since Figure 1 is a copy of the $D_{24}$ hole diagram (see [3]) this proves the result.

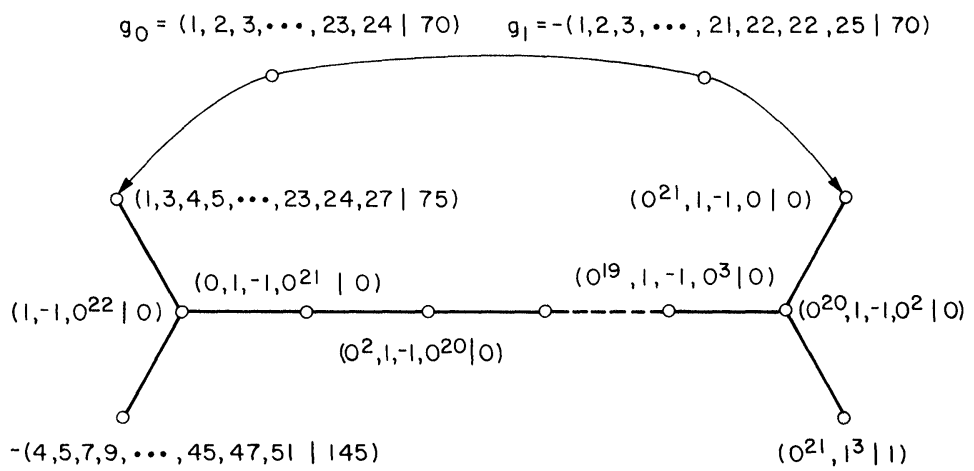

FIGURE 1. Hole diagram of type $D_{24}$ for Leech lattice.

Seidel ([7] ; see also Coxeter [4, p. 419] and Neumaier [5]) has recently remarked that elegant coordinates for the lattice $E_{8}$ may be obtained by considering the points of $Z^{9,1}$ orthogonal to the isotropic vector

$$
w=(1,1,1,1,1,1,1,1,1 \mid 3)
$$

Received by the editors August 11, 1981.

1980 Mathematics Subject Classification. Primary 10C05, 10E30.

(C) 1982 American Mathematical Society 0273-0979/81/0000-0331/\$01.75 
modulo multiples of $w$. It is easy to see that we obtain the Leech lattice in a similar way from the isotropic vector

$$
w=(1,3,5, \ldots, 45,47,51 \mid 145)
$$

in $\mathbf{Z}^{25,1}$.

However still more elegant coordinates may be obtained as follows. We consider the even unimodular lattice $L$ in $\mathbf{R}^{25,1}$ consisting of the points $\left(x_{0}, \ldots, x_{24} \mid x_{70}\right)$ where the $x_{i}$ are all in $\mathrm{Z}$ or all in $\mathrm{Z}+1 / 2$, and $x_{0}+x_{1}$ $+\cdots+x_{24}-x_{70}$ is in $2 Z$. (Up to isomorphism there is a unique even unimodular lattice in $\mathbf{R}^{25,1}$.) We assert that the Leech lattice can be regarded as the set of all vectors of $L$ orthogonal to

$$
w=(0,1,2, \ldots, 23,24 \mid 70)
$$

taken modulo multiples of $w$. Again it is convenient to work in a parallel hyperplane, the hyperplane $H=\{v \in L: v \cdot w=-1\}$. Figure 2 shows a set of vectors in this hyperplane which contains the $A_{24}$ hole diagram, and thus proves that $H \cap L$ is a copy of the Leech lattice.

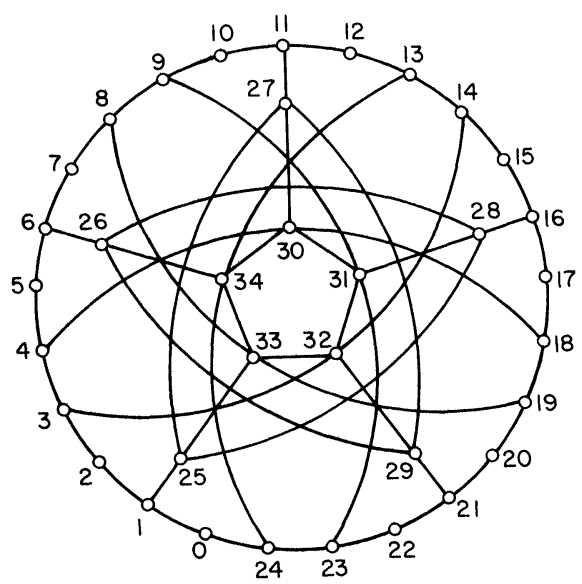

FIGURE 2. A portion of the Leech lattice contained in $H \cap L$. The coordinates of the points are as follows: $i(0 \leqslant i \leqslant 23):\left(0^{i},+1,-1,0^{23-i} \mid 0\right)$;

24: $\left(-\frac{1}{2}, \frac{1}{2}^{23}, \frac{3}{2} \mid \frac{5}{2}\right) ; 25:\left(-1^{2}, 0^{23} \mid 0\right) ; 26:\left(0^{7}, 1^{18} \mid 4\right)$

27: $\left(\frac{1}{2}^{12}, \frac{3}{2}^{13} \mid \frac{9}{2}\right) ; 28:\left(\frac{1}{2}^{17}, \frac{3}{2}^{8} \mid \frac{9}{2}\right) ; 29:\left(0^{22}, 1^{3} \mid 1\right)$;

30: $\left(0^{5}, 1^{14}, 2^{6} \mid 6\right) ; 31:\left(0^{10}, 1^{14}, 2 \mid 4\right) ; 32:\left(0^{4}, 1^{11}, 2^{10} \mid 7\right)$;

33: $\left(\frac{1}{2}^{9}, \frac{3}{2}^{11}, \frac{5}{2}^{5} \mid \frac{15}{2}\right) ; 34:\left(0^{14}, 1^{11} \mid 3\right)$. 
The representatives chosen for the points $v$ in Figure 2 have $v \cdot v=2$, which seems to provide the simplest coordinates. However each vector in $H$ has a unique isotropic representative modulo multiples of $w$, and using the results of [3] we have shown that

(i) if $v$ is any isotropic vector of $H \cap L$ then $v^{\perp} / v$ is another copy of the Leech lattice, and

(ii) if $v$ is the isotropic representative of the center of a deep hole in $H \cap L$ then $v^{\perp} / v$ is a copy of the Niemeier lattice $([2,3,6])$ corresponding to that hole.

For instance the sum of the vectors on the 25-gon visible in Figure 2 is $\left(1 / 2^{25} \mid 5 / 2\right)$, which is isotropic and proportional to the center of the corresponding hole of type $A_{24}$ in the Leech lattice $H \cap L$. Thus, for $v=\left(1^{25} \mid 5\right), v^{\perp} / v$ is the Niemeier lattice of type $A_{24}$. This result would not be affected if, when forming $v^{\perp}$, we replaced $L$ by the odd unimodular lattice $Z^{25,1}$.

In a similar way we have shown that inside $Z^{25,1}$ the vectors

$$
\begin{aligned}
& v_{1}=\left(1^{8}, 3^{9}, 5^{8} \mid 17\right), \quad v_{2}=\left(1^{13}, 3^{12} \mid 11\right), \\
& v_{3}=\left(1^{18}, 3^{7} \mid 9\right), \quad v_{4}=\left(1^{15}, 3^{9}, 5 \mid 11\right),
\end{aligned}
$$

have $v_{i}^{\perp} / v_{i}$ equal to the Niemeier lattices of types $A_{8}^{3}, A_{12}^{2}, A_{17} E_{7}, A_{15} D_{9}$ respectively, and many other examples can be produced at will.

\section{REFERENCES}

1. J. H. Conway, R. A. Parker and N. J. A. Sloane, The covering radius of the Leech lattice, Proc. Roy.Soc. (to appear)

2. J. H. Conway and N. J. A. Sloane, On the enumeration of lattices of determinant one, J. Number Theory (to appear).

appear).

3. - Twenty-three constructions for the Leech lattice, Proc. Roy. Soc. (to

4. H. S. M. Coxeter, Extreme forms, Canad. J. Math. 3 (1951), 391-441.

5. A. Neumaier, Lattices of Leech type, SIAM J. Algebraic und Discrete Methods (to appear).

6. H. V. Neimeier, Definite quadratische Formen der Dimension 24 and Diskriminante 1, J. Number Theory 5 (1973), 142-178.

7. J. A. Seidel, Discrete hyperbolic geometry, presented at Discrete Geometry Meeting, Mathematisches Forschungsinstitut, Oberwolfach, Germany, July 16, 1981.

DEPARTMENT OF PURE MATHEMATICS AND MATHEMATICAL STATISTICS, UNIVERSITY OF CAMBRIDGE, CAMBRIDGE CB2 1SB, ENGLAND

MATHEMATICS AND STATISTICS RESEARCH CENTER, BELL LABORATORIES, MURRAY HILL, NEW JERSEY 07974 